\title{
Inverse Design of Low-Boom Supersonic Concepts Using Reversed Equivalent-Area Targets*
}

\author{
$\mathrm{Wu} \mathrm{Li}{ }^{1}$ \\ NASA Langley Research Center, Hampton, Virginia 23681, USA \\ Sriram Rallabhandi ${ }^{2}$ \\ National Institute of Aerospace, Hampton, Virginia 23666, USA
}

\begin{abstract}
A promising path for developing a low-boom configuration is a multifidelity approach that (1) starts from a low-fidelity low-boom design, (2) refines the low-fidelity design with computational fluid dynamics (CFD) equivalent-area $\left(A_{\mathrm{e}}\right)$ analysis, and (3) improves the design with sonic-boom analysis by using CFD off-body pressure distributions. The focus of this paper is on the third step of this approach, in which the design is improved with sonicboom analysis through the use of CFD calculations. A new inverse design process for offbody pressure tailoring is formulated and demonstrated with a low-boom supersonic configuration that was developed by using the mixed-fidelity design method with CFD $A_{e}$ analysis. The new inverse design process uses the reverse propagation of the pressure distribution $(d p / p)$ from a mid-field location to a near-field location, converts the near-field $d p / p$ into an equivalent-area distribution, generates a low-boom target for the reversed equivalent area $\left(A_{e, r}\right)$ of the configuration, and modifies the configuration to minimize the differences between the configuration's $A_{\mathrm{e}, \mathrm{r}}$ and the low-boom target. The new inverse design process is used to modify a supersonic demonstrator concept for a cruise Mach number of 1.6 and a cruise weight of $30,000 \mathrm{lb}$. The modified configuration has a fully shaped ground signature that has a perceived loudness (PLdB) value of 78.5, while the original configuration has a partially shaped aft signature with a PLdB of 82.3.
\end{abstract}

\section{Nomenclature}

A $\quad=$ amplitude of oscillation

$A_{\mathrm{e}} \quad=$ classical equivalent area defined by Mach angle cut method

$A_{\mathrm{e}, \mathrm{r}} \quad=$ equivalent area defined by using reverse propagation of off-body pressure

BL = body length of the configuration or the aircraft concept

$C_{v} \quad=$ dimensionless dispersion parameter

$c_{0} \quad=$ ambient speed of sound

$M \quad=$ Mach number

$d p / p=($ calculated pressure - free-stream pressure $) /($ free-stream pressure $)$

$P \quad=$ nondimensional pressure or waveform

$S \quad=$ ray tube areas

$x, y=$ coordinates of a point on the plane

$\alpha \quad=$ relaxation factor, 0.5

$\varepsilon \quad=$ positive constant for regularization of diffusion equation

$\Gamma \quad=$ dimensionless thermoviscous parameter

$\rho_{0} \quad=$ ambient atmospheric density

$\sigma, \sigma_{k} \quad=$ nondimensional distance during propagation

$\tau=$ nondimensional time coordinate of the waveform

\footnotetext{
* AIAA Paper for the 29th AIAA Applied Aerodynamics Conference, June 2011, Honolulu, Hawaii.

${ }^{1}$ Senior Research Engineer, Aeronautics Systems Analysis Branch, Mail Stop 442.

${ }^{2}$ Senior Research Engineer, Resident at Aeronautics Systems Analysis Branch, Mail Stop 442.
} 
$\Delta \tau=$ a constant gap between two nondimensional time coordinates

$\theta_{v} \quad=$ dimensionless relaxation time parameter

\section{Introduction}

$\mathrm{D}$ EVELOPING a configuration with a shaped ground signature that is propagated from computational fluid dynamics (CFD) off-body pressure distributions is still a difficult task. A promising path for developing a lowboom configuration is a multifidelity approach that (1) starts from a low-fidelity, low-boom design, (2) refines the low-fidelity design with CFD equivalent-area $\left(A_{\mathrm{e}}\right)$ analysis, and (3) improves the design with sonic-boom analysis by using CFD off-body pressure distributions. Steps (1) and (2), along with the analysis portion of step (3), have been implemented in ModelCenter ${ }^{1}$ as a mixed-fidelity design process for low-boom supersonic concepts. ${ }^{2}$ Case studies using this mixed-fidelity process have been documented in Refs. 2 and 3. The main focus of this paper is on the development of an inverse design process for step (3) of the multifidelity design approach. A case study demonstrates how to tailor the off-body pressure to obtain a low-boom configuration with a fully shaped ground signature.

The theory behind the new off-body pressure tailoring is based on a reversed equivalent area $\left(A_{\mathrm{e}, \mathrm{r}}\right)$ that represents an accurate far-field approximation of the aircraft as a body of revolution. The $A_{\mathrm{e}, \mathrm{r}}$ is calculated from a mid-field pressure distribution in the following manner: the mid-field pressure distribution is reversely propagated to a near-field location by using the augmented Burgers equation and then converted to the equivalent-area distribution by using classical far-field theory. By using $A_{\mathrm{e}, \mathrm{r}}$, one can convert off-body pressure target matching to $A_{\mathrm{e}, \mathrm{r}}$ target matching. The new inverse design process starts with calculation of $A_{\mathrm{e}, \mathrm{r}}$ for a given configuration, generates a lowboom target for the configuration's $A_{\mathrm{e}, \mathrm{r}}$, and modifies the shape of the configuration to minimize the differences between the configuration's $A_{\mathrm{e}, \mathrm{r}}$ and the low-boom target.

A design case is used to demonstrate that the $A_{\mathrm{e}, \mathrm{r}}$ target matching problem can be solved with volume $A_{\mathrm{e}}$ shaping and shape optimization for lift tailoring with three twist angles of the horizontal tail. In this paper, the mid-field location is 3BL (or $381 \mathrm{ft}$ ) below the aircraft, and the near-field location is $50 \mathrm{ft}$ below the aircraft. The baseline configuration was originally designed by using the mixed-fidelity design process ${ }^{2}$ for a cruise weight of $30,000 \mathrm{lb}$, a cruise Mach number of 1.6, and a cruise altitude of 45,000 ft. Sonic-boom analysis with CFD off-body pressure distributions was used to verify that this mixed-fidelity design had a ground signature with a perceived loudness of 82.3 PLdB. After the $A_{\mathrm{e}, \mathrm{r}}$ target matching, the ground signature of the modified configuration is fully shaped and has a perceived loudness of 78.5 PLdB.

In the literature, there were many papers on design methods for developing a supersonic configuration with a shaped ground signature. See Ref. 4 for a survey and Refs. 5-10 for some recent advances on the development of low-boom design methods. So far, there is no published reference on any demonstration of using a design method to generate a low-boom supersonic configuration that has a fully shaped ground signature.

This paper is organized as follows. Section II introduces the augmented Burgers equation for reverse propagation, as well as its solution. In section III, the relationships among $A_{\mathrm{e}, \mathrm{r}}, A_{\mathrm{e}}$, and the mid-field pressure distribution are quantified by examples. The design case is documented in section IV, and concluding remarks are given in section $\mathrm{V}$.

\section{Augmented Burgers Equation for Reverse Propagation}

The augmented Burgers equation is used for propagation of the off-body pressure distributions, as well as the equivalent-area or $F$-function distributions. ${ }^{11}$ This section describes the augmented Burgers equation and its solution method for reversely propagating the mid-field pressure distributions to a near-field location. The reverse propagation method yields a more accurate equivalent-area approximation of an aircraft for low-boom design than the traditional $A_{\mathrm{e}}$ (see section III).

\section{A. Augmented Burgers Equation}

The augmented Burgers equation includes effects such as nonlinearity, thermoviscous absorption, and molecular relaxation phenomena during the propagation of pressure distributions through the atmosphere. The dimensionless Burgers equation is shown in Eq. (1), where the last two terms represent geometrical spreading and propagation through a stratified atmosphere. 


$$
\frac{\partial P}{\partial \sigma}=P \frac{\partial P}{\partial \tau}+\frac{1}{\Gamma} \frac{\partial^{2} P}{\partial \tau^{2}}+\sum_{v} C_{v} \frac{\frac{\partial^{2}}{\partial \tau^{2}}}{1+\theta_{v} \frac{\partial}{\partial \tau}} P-\frac{\frac{\partial}{\partial \sigma} S}{2 S} P+\frac{\frac{\partial}{\partial \sigma}\left(\rho_{0} c_{0}\right)}{2 \rho_{0} c_{0}} P
$$

As the initial waveform, for which the nondimensional version is $P(0, \tau)$, moves downward toward the ground, the nondimensional distance $\sigma$ from the waveform to the aircraft increases. The waveform is nondimensionalized by using a reference pressure $P_{\text {ref. }}$. The shock formation distance of a plane wave with amplitude $P_{\text {ref }}$ is used to nondimensionalize the actual distance. The dimensionless time $\tau$ is a function of the arrival time of the waveform, at a fixed altitude, for a stationary observer. The dimensionless thermoviscous parameter $\Gamma$, the dimensionless relaxation time parameter $\theta_{v}$, the dimensionless dispersion parameter $C_{v}$, the ray tube area $S$, the atmospheric density $\rho_{0}$, and the speed of sound $c_{0}$ are obtained under the assumption of a standard atmosphere. A ray tracing model, which computes the ray tube area as well as the atmosphere-related parameters such as molecular relaxation and absorption values, is included in the propagation process. The initial waveform is then propagated toward the ground or any mid-field location iteratively for a finite number of shock formation distances: $0=\sigma_{0}<\sigma_{1}<\cdots<\sigma_{\mathrm{m}}=$ the ground or the mid-field location.

The augmented Burgers equation can be modified for the reverse propagation of the waveform as

$$
\frac{\partial P}{\partial \sigma}=-P \frac{\partial P}{\partial \tau}-\frac{1}{\Gamma} \frac{\partial^{2} P}{\partial \tau^{2}}-\sum_{v} C_{v} \frac{\frac{\partial^{2}}{\partial \tau^{2}}}{1+\theta_{v} \frac{\partial}{\partial \tau}} P-\frac{\frac{\partial}{\partial \sigma} S}{2 S} P+\frac{\frac{\partial}{\partial \sigma}\left(\rho_{0} c_{0}\right)}{2 \rho_{0} c_{0}} P
$$

In contrast to Eq. (1), $\sigma=0$ in Eq. (2) corresponds to the waveform at the initial mid-field location, and as $\sigma$ increases, the waveform moves toward the aircraft.

\section{B. Numerical Solution of Augmented Burgers Equation for Reverse Propagation}

While nonlinear effects during propagation steepen the waveform and impose an upper bound on the allowed spatial step size during iterations, reversing the nonlinear propagation smoothens the waveform and poses no numerical difficulty or spatial step size restriction. The absorption and the relaxation in the reverse direction increase the magnitude of the waveform rather than attenuate it, as would occur when these are applied in the forward propagation. The same ray tracing routine for the forward propagation is used for the reverse propagation, except that the direction of propagation is reversed.

As in the case of the forward propagation process, the reverse propagation is solved by using an operator splitting technique. If both the time-step size $\Delta \tau$ and the spatial step size $\left(\sigma_{k}-\sigma_{k-1}\right)$ are small enough, then the error that is induced by such a splitting methodology is insignificant. The reverse nonlinear equation is

$$
\frac{\partial P}{\partial \sigma}=-\frac{\partial\left(\frac{p^{2}}{2}\right)}{\partial \tau}
$$

Equation (3) is solved with an upwind flux-splitting method that uses the following formulas:

$$
\begin{gathered}
\frac{P_{\mathrm{nl}, i}^{k}-P_{i}^{k-1}}{\sigma_{k}-\sigma_{k-1}}=-\frac{f_{i+\frac{1}{2}}^{k-1}-f_{i-\frac{1}{2}}^{k-1}}{\Delta \tau} \\
f_{i+\frac{1}{2}}^{k-1}= \begin{cases}\frac{1}{2}\left[P_{i}^{k-1}\right]^{2} & \text { if } P_{i}^{k-1}+P_{i+1}^{k-1}>0 \\
\frac{1}{2}\left[P_{i+1}^{k-1}\right]^{2} & \text { if } P_{i}^{k-1}+P_{i+1}^{k-1} \leq 0\end{cases} \\
f_{i-\frac{1}{2}}^{k-1}= \begin{cases}\frac{1}{2}\left[P_{i-1}^{k-1}\right]^{2} & \text { if } P_{i-1}^{k-1}+P_{i}^{k-1}>0 \\
\frac{1}{2}\left[P_{i}^{k-1}\right]^{2} & \text { if } P_{i-1}^{k-1}+P_{i}^{k-1} \leq 0\end{cases}
\end{gathered}
$$

In the above equations, the symbol $\sigma_{k}$ denotes the next altitude location in reverse propagation, $\Delta \tau$ is the constant distance between two dimensionless time values for all $\sigma, P_{i}^{k-1}=P\left(\sigma_{k-1}, i \cdot \Delta \tau\right)$, and $P_{\mathrm{nl}, i}^{k} \approx P\left(\sigma_{k}, i \cdot \Delta \tau\right)$. Note that Eq. (4) computes $P_{\mathrm{nl}, i}^{k}$ explicitly from the known data. 
Absorption is modeled as a diffusion equation, while relaxation is modeled as a diffusion equation with a mixedderivative term. The reverse diffusion equation is inherently ill-posed, which makes Eq. (2) difficult to solve numerically. Several regularization techniques can be used to develop a reverse propagation algorithm that includes absorption and relaxation effects. We studied the pseudo-parabolic and quasi-reversible regularization schemes ${ }^{12}$ that are given in Eqs. (5) and (6), respectively.

$$
\begin{aligned}
& \frac{\partial P}{\partial \sigma}=-A \frac{\partial^{2} P}{\partial \tau^{2}}-\varepsilon \cdot A \frac{\partial^{2}}{\partial \tau^{2}}\left(\frac{\partial P}{\partial \sigma}\right) \\
& \frac{\partial P}{\partial \sigma}=-A \frac{\partial^{2} P}{\partial \tau^{2}}-\varepsilon \cdot A \frac{\partial^{2}}{\partial \tau^{2}}\left(\frac{\partial^{2} P}{\partial \tau^{2}}\right)
\end{aligned}
$$

In the above two equations, the original diffusion equation is modified by the last term to stabilize the numerical solution of the modified equation at the expense of adding numerical error in the solution. The constant $\varepsilon$ is a positive scalar that makes Eqs. (5) and (6) numerically solvable. A desirable value of $\varepsilon$ (i.e., $10^{-4}$ for the reverse propagation calculation in this paper) is determined by a trial-and-error method: $\varepsilon$ must be large enough that the numerical solution of the modified equation can be generated, and $\varepsilon$ must be small enough that the solution of the modified equation is an accurate approximation of the original diffusion equation. During the course of the evaluation and verification, the pseudo-parabolic scheme was shown to be better than the quasi-reversible scheme in terms of stability and accuracy. Therefore, all of the reverse propagation results in this paper are generated with the pseudo-parabolic scheme.

The vector $P_{\mathrm{nl}}^{k}$ that is defined by Eq. (4) is a solution of the reversed nonlinear equation and can be updated by using the following linear equations to include the absorption and relaxation effects:

$$
\begin{gathered}
A_{\mathrm{mr}}\left(\theta_{v, \mathrm{ox}}, C_{v, \mathrm{ox}}\right) \cdot P_{\mathrm{ox}}^{k}=B_{\mathrm{mr}}\left(\theta_{v, \mathrm{ox}}, C_{v, \mathrm{ox}}\right) \cdot P_{\mathrm{nl}}^{k} \\
A_{\mathrm{mr}}\left(\theta_{v, \mathrm{ni}}, C_{v, \mathrm{ni}}\right) \cdot P_{\mathrm{ni}}^{k}=B_{\mathrm{mr}}\left(\theta_{v, \mathrm{ni}}, C_{v, \mathrm{ni}}\right) \cdot P_{\mathrm{ox}}^{k} \\
A_{\mathrm{tvr}} \cdot P_{\mathrm{tvr}}^{k}=B_{\mathrm{tvr}} \cdot P_{\mathrm{ni}}^{k} \\
P^{k}=\lambda_{\mathrm{rst}} \cdot P_{\mathrm{tvr}}^{k}
\end{gathered}
$$

Equation (7) represents the reversed molecular relaxation by oxygen, Eq. (8) represents the reversed molecular relaxation by nitrogen, and Eq. (9) represents the reversed thermoviscous absorption. The parameters $\theta_{v, 0 x}, C_{v, \text { ox }}, \theta_{v, \text { ni }}$, and $C_{\mathrm{v}, \mathrm{ni}}$ are dimensionless relaxation time parameters and dispersion parameters for oxygen and nitrogen, respectively. The tridiagonal matrices $A_{\mathrm{mr}}\left(\theta_{v, \mathrm{ox}}, C_{v, \mathrm{ox}}\right), B_{\mathrm{mr}}\left(\theta_{v, \mathrm{ox}}, C_{v, \mathrm{ox}}\right), A_{\mathrm{mr}}\left(\theta_{v, \mathrm{ni}}, C_{v, \mathrm{ni}}\right), B_{\mathrm{mr}}\left(\theta_{v, \mathrm{ni}}, C_{v, \mathrm{ni}}\right), A_{\mathrm{tvr}}$, and $B_{\mathrm{tvr}}$ are defined in the appendix. The final iterative waveform solution $P^{k}$ for the reverse propagation at the altitude location $\sigma_{k}$ is given in Eq. (10). The constant $\lambda_{\text {rst }}$ is determined by using the ray tracing method and accounts for the ray tube spreading and stratification in the reverse direction.

The reverse propagation process starts with the waveform $P_{i}^{0}=P(0, i \cdot \Delta \tau)$ at the initial mid-field location; then the waveform $P^{k}$ is computed at the altitude location $\sigma_{k}$ via the intermediate waveform solutions $P_{\mathrm{nl}}^{k}, P_{\mathrm{ox}}^{k}, P_{\mathrm{ni}}^{k}$, and $P_{\mathrm{tvr}}^{k}$, for $k=1,2, \ldots$. The reverse propagation process terminates when $\sigma_{k}$ reaches the desired near-field location.

\section{Equivalent Area Defined by Reverse Propagation}

By using the reverse propagation method that is discussed in the previous section, a pressure distribution at a mid-field location can be reversely propagated to a near-field location. The near-field $d p / p$ can be converted back to an equivalent-area distribution, which is called the reversed equivalent area $A_{\mathrm{e}, \mathrm{r}}$, by using the following equation:

$$
A_{e, r}(x)=4 \cdot \frac{\left(2 \cdot R \cdot \sqrt{M^{2}-1}\right)^{\frac{1}{2}}}{\gamma \cdot M^{2}} \int_{0}^{x}(d p / p)\left(t-x_{0}\right) \cdot \sqrt{x-t} d t
$$

In Eq. (11), $M$ is the cruise Mach number, $R$ is the distance from the near-field location of the $d p / p$ to the aircraft, $x_{0}$ is the starting location of the nonzero segment of the near-field $d p / p$, and $\gamma=1.4$. Equation (11) is a composition of 
the relationship between the $F$-function and $d p / p$ (see Eq. (1) in Ref. 13) and the conversion from $F$-function to equivalent area (see Eq. (3) in Ref. 14). In this paper, $A_{\mathrm{e}, \mathrm{r}}$ is always defined by Eq. (11) and the reverse propagation of the off-body pressure at 3BL below the aircraft to the near-field location $R=50 \mathrm{ft}$.

In traditional far-field theory, $A_{\mathrm{e}}$ accounts only for the volume (monopole) and lift (dipole) effects, with the assumption that the higher order effects are insignificant for sonic-boom analysis. However, the far-field theory assumption is invalid for complex, non-slender configurations. The following example quantifies the inaccuracy of this assumption for a wing-body configuration.

Figure 1 shows that the body of revolution that is defined by $A_{\mathrm{e}}$ generates a $d p / p$ at $3 \mathrm{BL}$ that is significantly different from the $d p / p$ of the three-dimensional configuration for $80 \leq x \leq 100$. The two signatures for the CFD $A_{\mathrm{e}}$ and $d p / p$, respectively, are also different, as shown in Fig. 2 . The $d p / p$ at $3 \mathrm{BL}$ below the configuration is computed by using Cart $3 \mathrm{D}^{15}$ with a rotated and stretched volume grid of 20 million cells. ${ }^{16}$

In contrast, the reversed equivalent area is a highly accurate representation of the three-dimensional configuration for sonic-boom analysis. Figures 3 and 4 show that $A_{\mathrm{e}, \mathrm{r}}$ has an almost identical $d p / p$ at $3 \mathrm{BL}$ and the same ground signature as the wing-body configuration when the CFD off-body $d p / p$ distribution is used for sonicboom analysis.

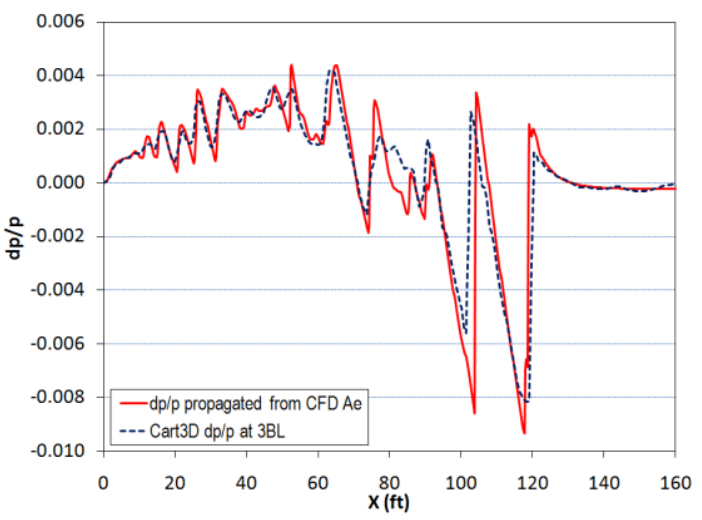

Figure 1. $d p / p$ for wing-body configuration and CFD $A_{\mathrm{e}}$.

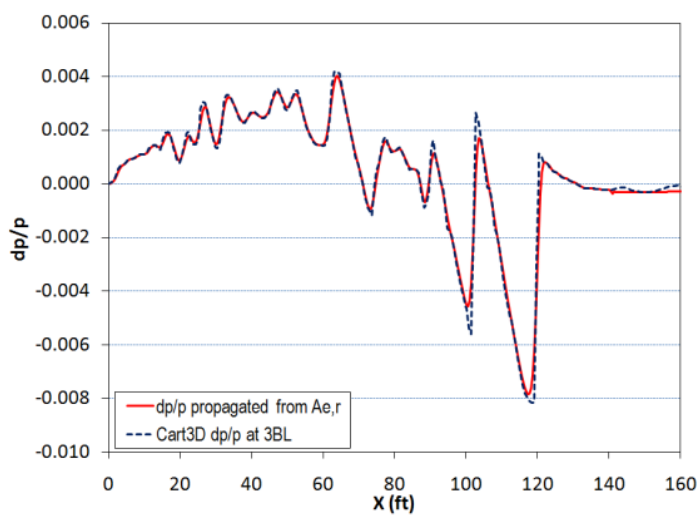

Figure 3. $d p / p$ for wing-body configuration and $A_{\mathrm{e}, \mathrm{r}}$.

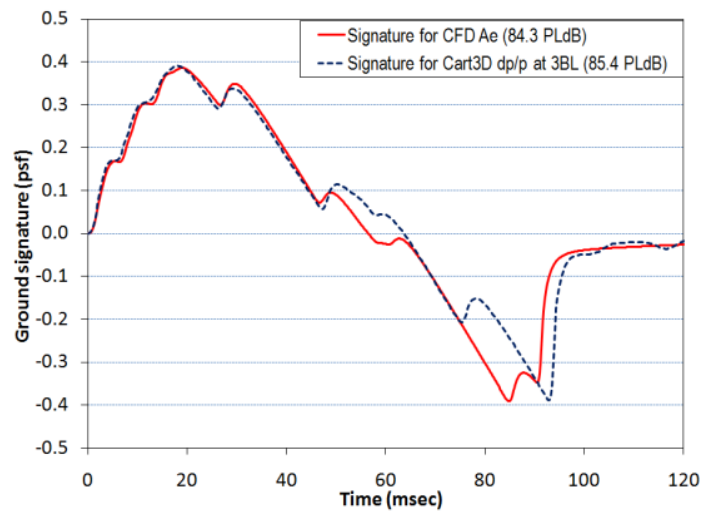

Figure 2. Ground signatures for $d p / p$ and $A_{\mathrm{e}}$.

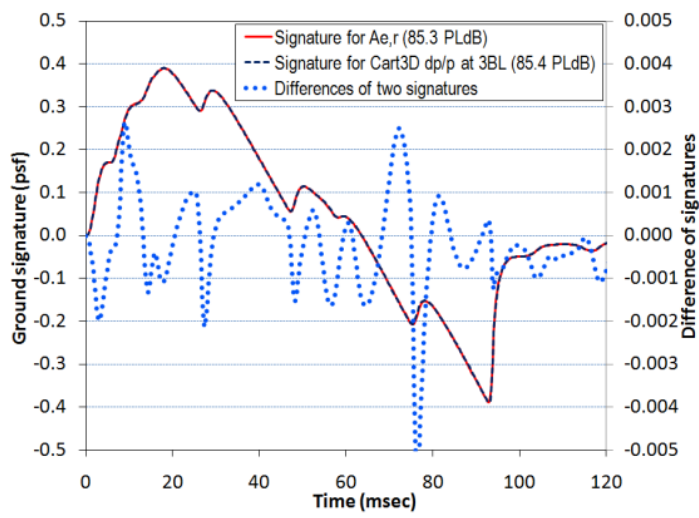

Figure 4. Ground signatures for $d p / p$ and $A_{\mathrm{e}, \mathrm{r}}$.

The comparison shows good agreement between $A_{\mathrm{e}}$ and $A_{\mathrm{e}, \mathrm{r}}$ for $x \leq 94$ (see Fig. 5). The CFD surface pressure distributions (see Fig. 6) do not clearly indicate why $A_{\mathrm{e}}$ and $A_{\mathrm{e}, \mathrm{r}}$ become drastically different for $x>94$. Note that the end area value of $A_{\mathrm{e}}$ that is obtained from the Mach angle cuts of the CFD surface solution is completely dependent on the total lift, but the end area value of $A_{\mathrm{e}, \mathrm{r}}$ is affected by other three-dimensional flow properties, such as the pressure variations behind the aircraft. 


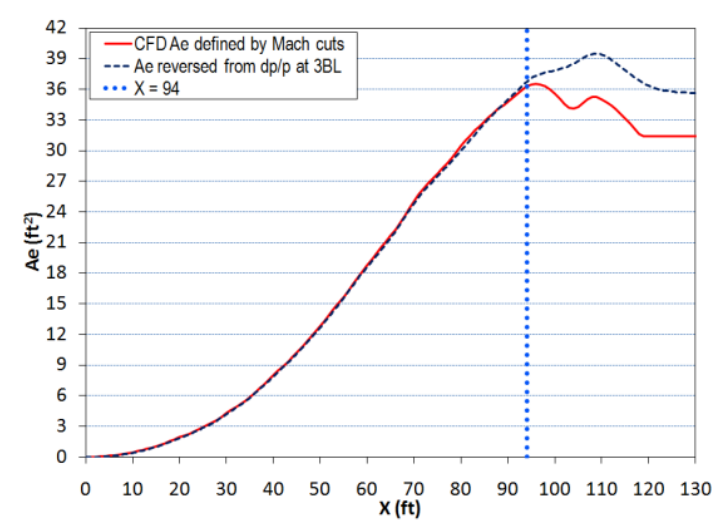

Figure 5. Comparison of $A_{\mathrm{e}}$ and $\boldsymbol{A}_{\mathrm{e}, \mathrm{r}}$.

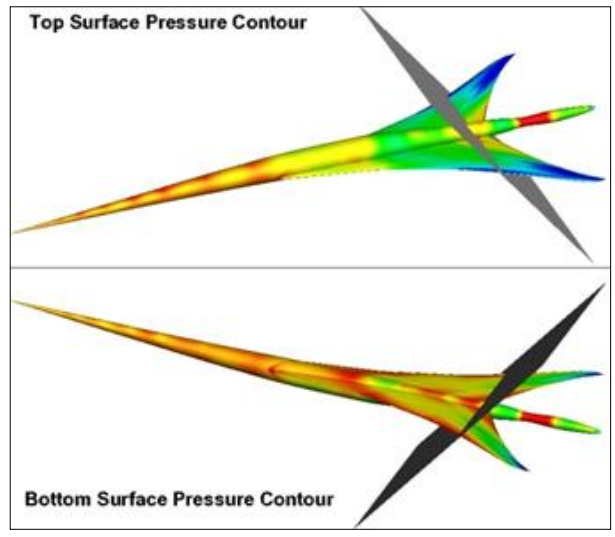

Figure 6. Mach angle cut at $X=94$.

\section{Matching Reversed Equivalent Area to a Target for Low-Boom Design}

The analysis in section III shows that the ground signatures for $d p / p$ at $3 \mathrm{BL}$ and $A_{\mathrm{e}, \mathrm{r}}$ are nearly identical for a wing-body configuration (see Fig. 4). In fact, the differences between the ground signatures for $d p / p$ at $3 \mathrm{BL}$ and $A_{\mathrm{e}, \mathrm{r}}$ are caused by two sources of numerical error-numerical error in the reverse propagation and in the conversion from $A_{\mathrm{e}, \mathrm{r}}$ to the near-field pressure in the forward propagation. These errors are insignificant and cause no significant difference between the two signatures. As a result, the problem of off-body pressure tailoring for lowboom design is equivalent to finding a configuration that has a low-boom $A_{\mathrm{e}, \mathrm{r}}$. In particular, we can design a lowboom configuration by matching its $A_{\mathrm{e}, \mathrm{r}}$ to a low-boom target.

The design process consists of fuselage/pod shaping for equivalent-area matching and design of experiments (DOE) for modification of the aft shape of $A_{\mathrm{e}, \mathrm{r}}$. The baseline configuration was originally designed by using a mixed-fidelity $A_{\mathrm{e}}$ matching process ${ }^{2}$ for a cruise weight of 30,000 lb, a cruise Mach number of 1.6, and a cruise altitude of 45,000 ft. (See Fig. 10 in Ref. 2 for the sonic-boom analysis of this configuration.) The same mixedfidelity design process that is given in Ref. 2 (except that $A_{\mathrm{e}}$ is replaced with $A_{\mathrm{e}, \mathrm{r}}$ ) is used here for volume shaping to tailor the overall shape of $A_{\mathrm{e}, \mathrm{r}}$, while a three-level full-factorial DOE for three twist angles of the horizontal tail is used to modify the aft shape of $A_{\mathrm{e}, \mathrm{r}}$.

To track the design progress, we used "B" to indicate the fuselage/pod shaping by $\mathrm{BOSS}^{4}$ and " $\mathrm{D}$ " to indicate the DOE runs. With this labeling system, D3B7 is the configuration that was obtained from the third DOE run of B7, while B7 is the configuration that was obtained from the seventh fuselage/pod shaping iteration of the baseline configuration. The final design is D8B1D3B7, which has a fully shaped ground signature and a PLdB of 76.6.

Each fuselage/pod shaping iteration that is performed with BOSS uses the following mixed-fidelity $A_{\mathrm{e}, \mathrm{r}}$ formula:

$$
A_{e, r}^{\text {mixed }}=A_{e, r}^{\text {old }}+A_{e, \text { new }}^{\text {volume }}-A_{e, \text { old }}^{\text {volume }}
$$

The mixed-fidelity formula does not require the calculation of $A_{\mathrm{e}, \mathrm{r}}$ for modified configurations during the BOSS optimization iterations, which makes the fuselage/pod shaping process efficient. The complete inverse design process to obtain D8B1D3B7 can be summarized as follows:

(a) Compute the $d p / p$ at $3 \mathrm{BL}$ below the configuration.

(b) Compute $A_{\mathrm{e}, \mathrm{r}}$ from $d p / p$ at $3 \mathrm{BL}$.

(c) Find a low-boom equivalent-area target that is as close to $A_{\mathrm{e}, \mathrm{r}}$ as possible.

(d) If the differences between the target and $A_{\mathrm{e}, \mathrm{r}}$ cannot be reduced by fuselage/pod volume modification, then go to step (g).

(e) Modify the fuselage or the pod so that $A_{e, r}^{\text {mixed }}$ is closer to the target configuration than $A_{\mathrm{e}, \mathrm{r}}\left(=A_{e, r}^{\text {old }}\right)$.

(f) Go back to step (a).

(g) Use DOE for three twist angles to reshape the horizontal tail. The objective function is the PLdB value of the ground signature for $d p / p$ at 3BL. Pick the modified configuration that has the lowest PLdB value.

(h) If the ground signature is not fully shaped, then go back to step (b). 
Note that the final design D8B1D3B7 is obtained when step $(\mathrm{h})$ is reached for the second time. During the inverse design process, the angle of attack for the Cart3D sonic-boom analysis was fixed at $0.5 \mathrm{deg}$; this value was determined by the baseline cruise weight of 30,000 lb. The low-boom targets were generated by a parametric equivalent-area target explorer. ${ }^{3}$

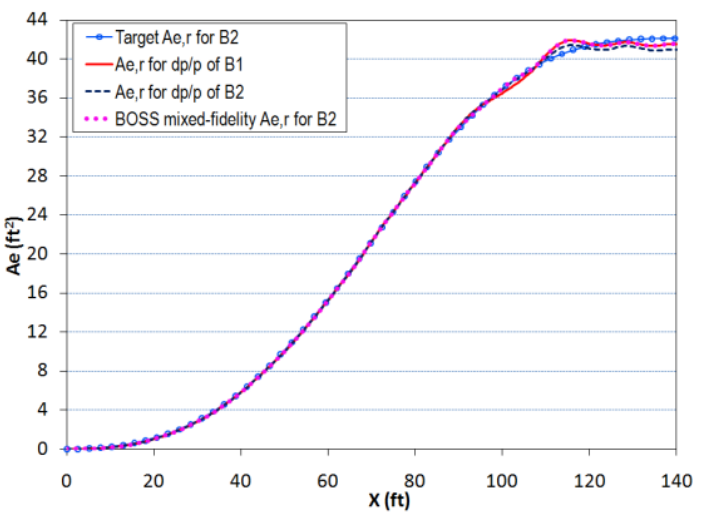

Figure 7. $A_{\mathrm{e}, \mathrm{r}}$ matching for $\mathrm{B} 2$ design.

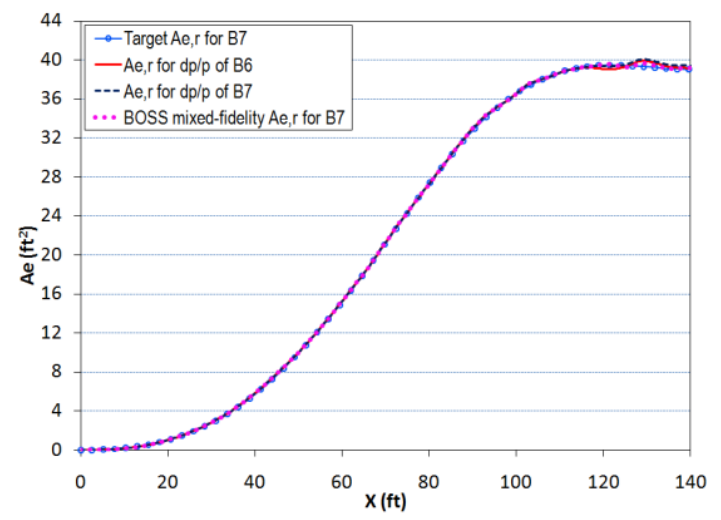

Figure 9. $A_{\mathrm{e}, \mathrm{r}}$ matching for $\mathrm{B} 7 \mathrm{design}$.

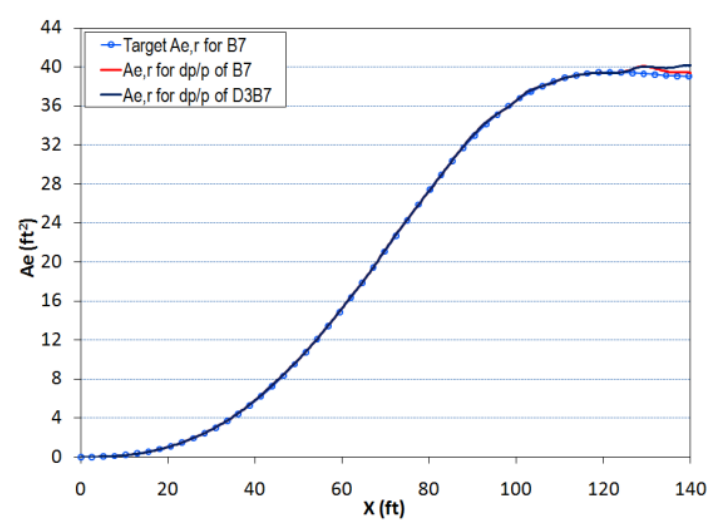

Figure 11. Comparison of $A_{\mathrm{e}, \mathrm{r}}$ for D3B7 design.

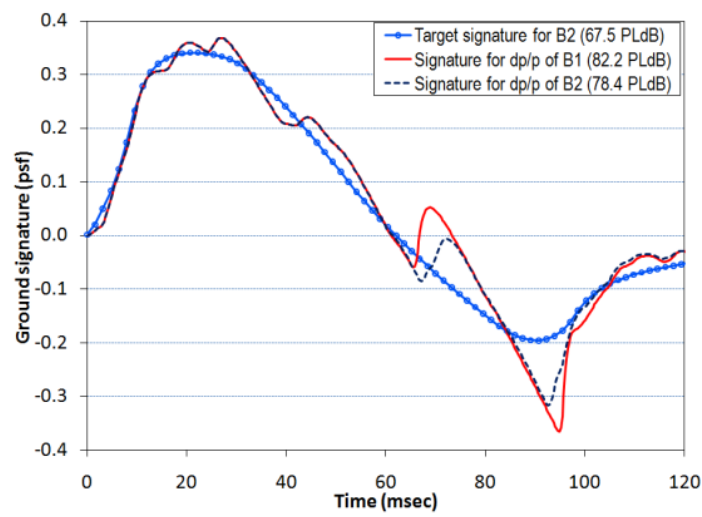

Figure 8. Comparison of ground signatures for $\mathrm{B} 2$ design.

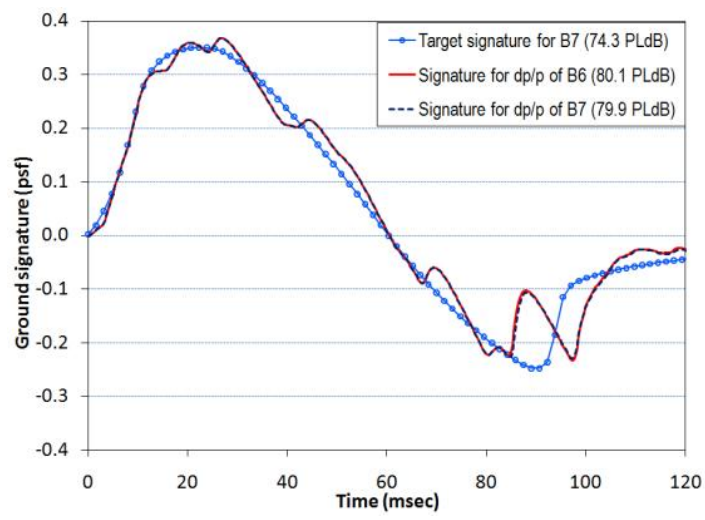

Figure 10. Comparison of ground signatures for $B 7$ design.

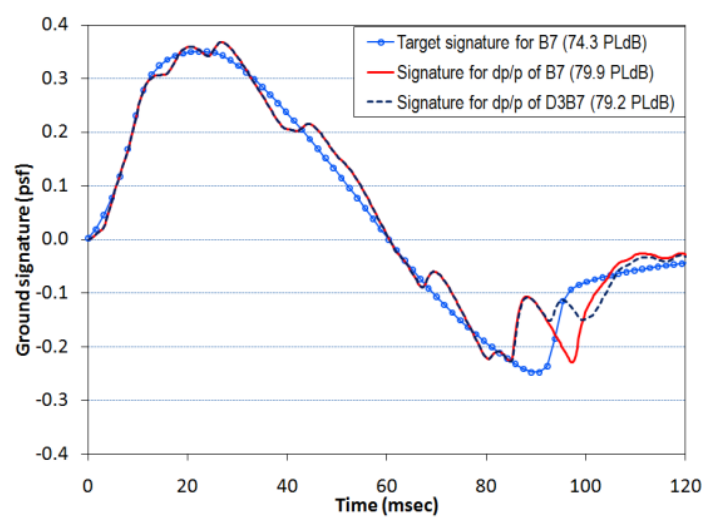

Figure 12. Comparison of ground signatures for D3B7 design.

After two fuselage shaping iterations, the gap between the $A_{\mathrm{e}, \mathrm{r}}$ of the configuration and the target for $X \leq 107$ was eliminated (see Fig. 7), which resulted in a reduction of 4 PLdB in the ground signature (see Fig. 8). Further fuselage shaping was performed until the aft shape of the ground signature was close to the sinusoidal shape. After five additional fuselage shaping iterations, the gap between the $A_{\mathrm{e}, \mathrm{r}}$ of the configuration and the target for $X \leq 125$ was eliminated (see Fig. 9); however, the PLdB value of the ground signature for the B7 design remained higher 
than that of the B2 design (see Fig. 10). The B7 design was accepted because the aft shape of its ground signature had a much lower peak overpressure and was less likely to be an $N$-wave than that of the B2 design. Each $A_{\mathrm{e}, \mathrm{r}}$ matching iteration typically required a new low-boom target.

The differences between the $A_{\mathrm{e}, \mathrm{r}}$ of the B7 design and the target were not affected by volume shaping. Thus, a DOE for three twist angles of the horizontal tail was used to further modify the aft shape of the ground signature. The limits of the twist angles for the root, midspan, and tip airfoils were -0.2 to $0.2,-0.4$ to 0.4 , and -1.0 to $1.0 \mathrm{deg}$, respectively. A three-level full-factorial DOE was performed with 27 configuration variations. The third DOE configuration, with twist angles of $0.2,-0.4$, and $-1.0 \mathrm{deg}$ for the root, midspan, and tip airfoils, respectively, was selected because of the aft shape of its ground signature (see Fig. 12). Figure 11 shows that $A_{\mathrm{e}, \mathrm{r}}$ was affected by the horizontal tail at $X \geq 130$.

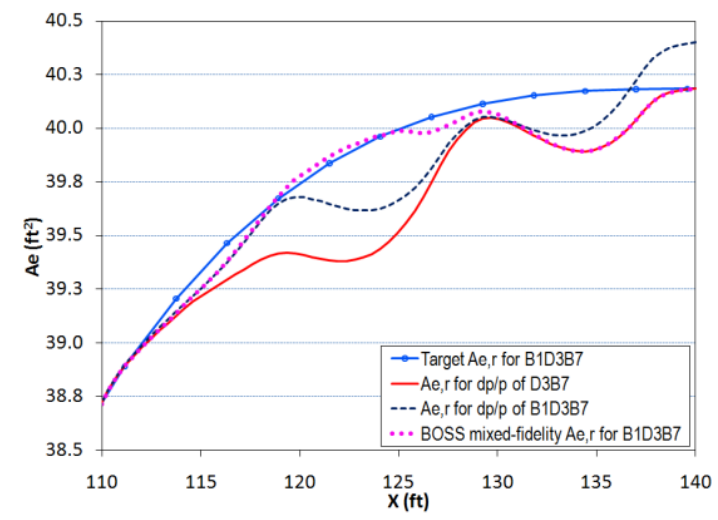

Figure 13. $A_{\mathrm{e}, \mathrm{r}}$ matching for B1D3B7 design.

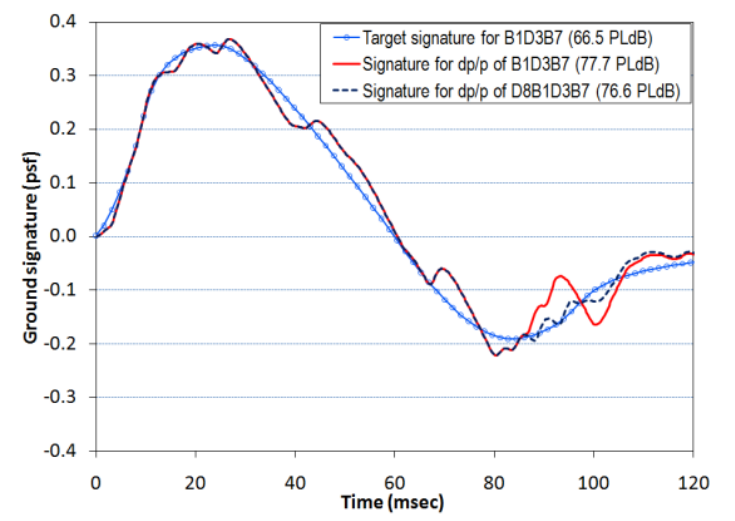

Figure 15. Comparison of ground signatures for D8B1D3B7 design.

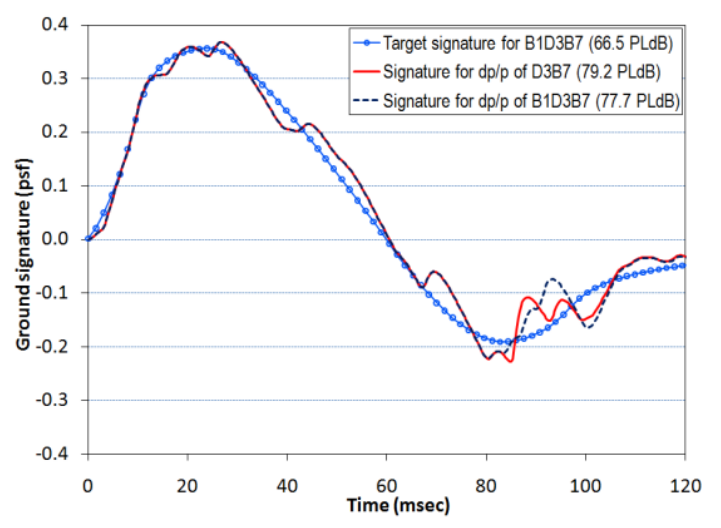

Figure 14. Comparison of ground signatures for B1D3B7 design.

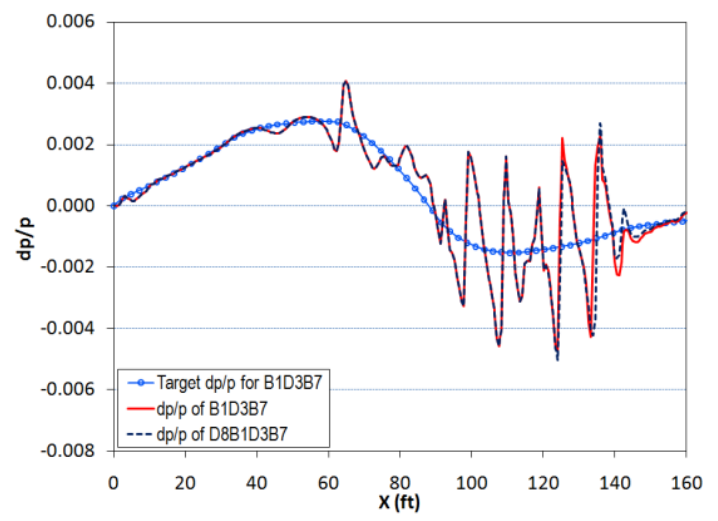

Figure 16. Change in $d p / p$ for D8B1D3B7 design.

Another $A_{\mathrm{e}, \mathrm{r}}$ target was generated for aft pod shaping. The pod shaping was intended to eliminate the differences between the $A_{\mathrm{e}, \mathrm{r}}$ of the configuration and the target for $117 \leq X \leq 129$, but the actual CFD analysis for $A_{\mathrm{e}, \mathrm{r}}$ shows that the differences for $117 \leq X \leq 135$ were only partially eliminated (see Fig. 13). The improvement in the aft shape of the ground signature is difficult to quantify, but a reduction of 1.5 PLdB in the perceived loudness of the ground signature makes the B1D3B7 design more desirable than the D3B7 design (see Fig. 14). Further fuselage and pod shaping did not yield any meaningful improvement of the ground signature. Another DOE for three twist angles was performed to tailor the aft shape of the ground signature. The same twist angle limits and DOE settings were used. The eighth DOE configuration, with twist angles of $-0.2,0.4$, and -1.0 deg for the root, midspan, and tip airfoils, respectively, was selected because of the nearly perfect aft shape of its ground signature (see Fig. 15). The minor differences that can be seen between the $d p / p$ distributions of the B1D3B7 and D8B1D3B7 designs demonstrate the sensitivity of the ground signature to the $d p / p$ at $3 \mathrm{BL}$ (see Fig. 16).

Because the angle of attack is fixed at 0.5 deg during the inverse design process, the signature for the D8B1D3B7 design shown in Fig. 15 is for a cruise weight of 27,800 lb. A sensitivity analysis of the ground 
signature for the D8B1D3B7 design with respect to the cruise weight is performed for a set of angles of attack. Figure 17 shows that the D8B1D3B7 design still has a fully shaped ground signature and a PLdB of 78.5 at the desired cruise weight of 30,000 lb; Fig. 18 shows that the shapes of all $A_{\mathrm{e}, \mathrm{r}}$ are similar. These two figures show the sensitivity of the ground signature and the $A_{\mathrm{e}, \mathrm{f}}$ for the D8B1D3B7 design with respect to cruise weight changes.

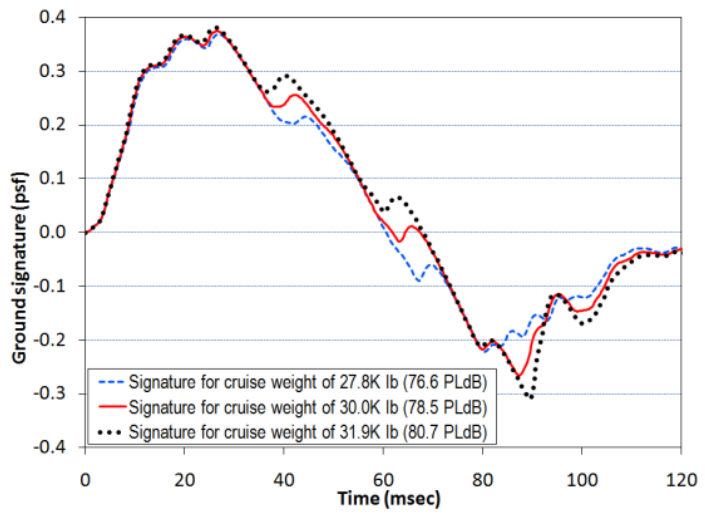

Figure 17. Comparison of ground signatures for different cruise weights.

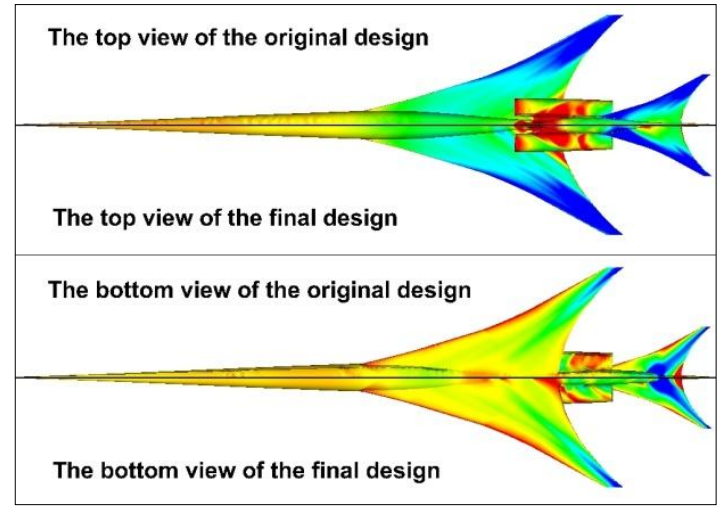

Figure 19. Surface $d p / p$ for original and final designs.

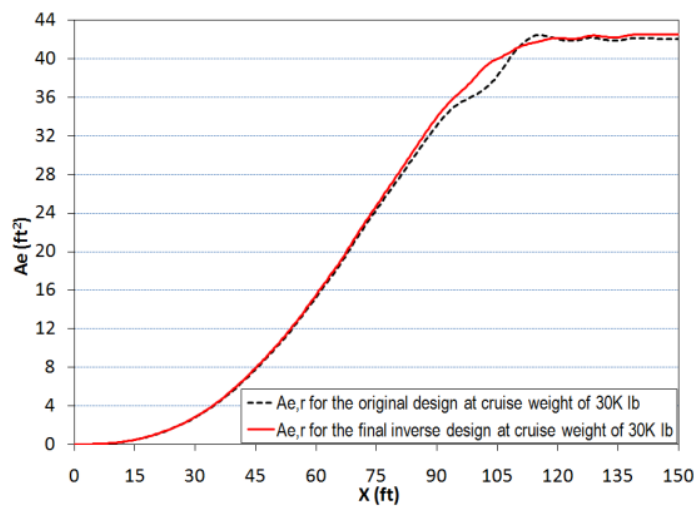

Figure 21. $A_{\mathrm{e}, \mathrm{r}}$ for original and final designs.

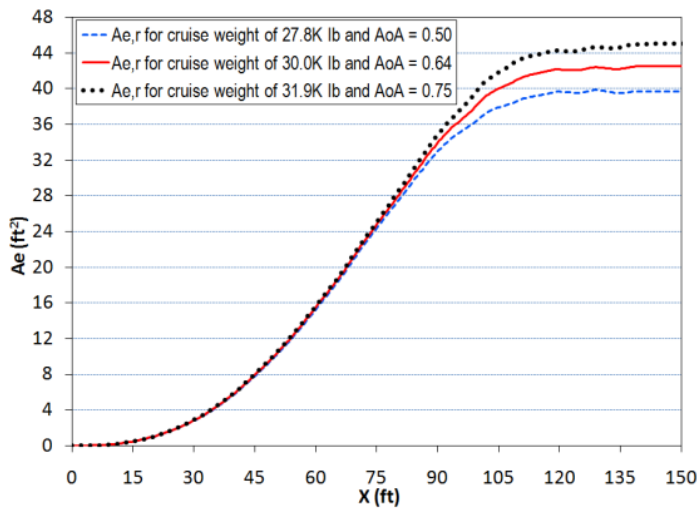

Figure 18. Comparison of $A_{\mathrm{e}, \mathrm{r}}$ for different cruise weights.

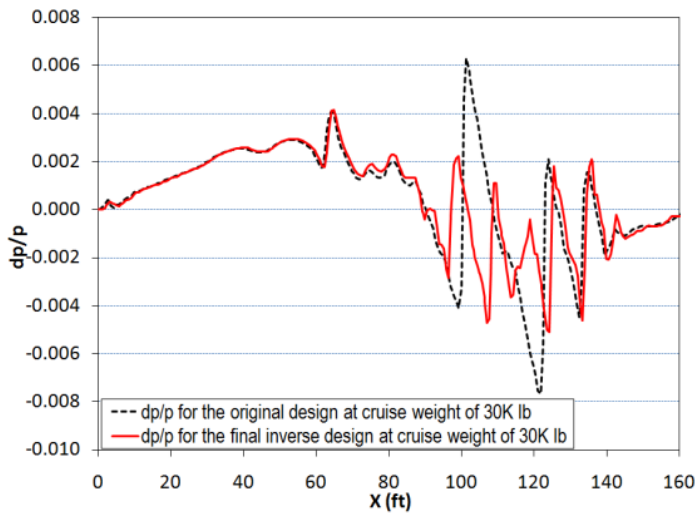

Figure 20. $d p / p$ at 3BL for original and final designs.

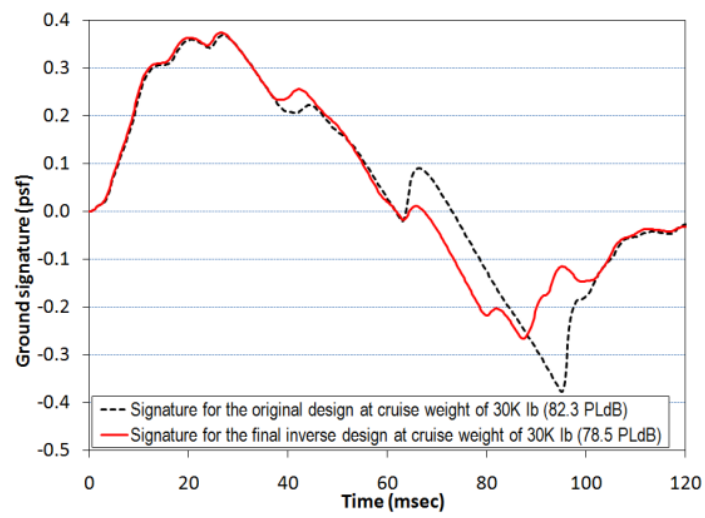

Figure 22. Ground signatures for original and final designs.

Figures 19-22 compare the surface pressure distributions, the $d p / p$ distributions at $3 \mathrm{BL}$, the $A_{\mathrm{e}, \mathrm{r}}$ values, and the ground signatures, respectively, for the original CFD $A_{\mathrm{e}}$ design (i.e., the baseline) and the final inverse design. The most noticeable differences in the surface pressure distributions of the two designs are on the trailing edge of the wing below the nacelle and on the horizontal tail (see Fig. 19). Note that the pressure differences on the lower surface of the wing could be caused by the combined effect of the fuselage shaping and the difference in the angles of attack for the two designs. 


\section{Concluding Remarks}

The augmented Burgers equation is used to propagate the computational fluid dynamics (CFD) pressure distribution $(d p / p)$ at a mid-field location to a near-field location. Then, the near-field $d p / p$ is converted to the reversed equivalent area $\left(A_{\mathrm{e}, \mathrm{r}}\right)$ by using classical far-field theory. The resulting $A_{\mathrm{e}, \mathrm{r}}$ contains much more information about the three-dimensional flow for sonic-boom analysis and has almost the same ground signature as the threedimensional configuration when analyzed by propagation of the mid-field pressure distribution. As a result, the problem of tailoring a mid-field pressure distribution for a low-boom design can be solved by matching $A_{\mathrm{e}, \mathrm{r}}$ to a lowboom target.

Matching the $A_{\mathrm{e}, \mathrm{r}}$ to a low-boom target has three clear advantages over tailoring a mid-field $d p / p$ : (1) the differences between the $A_{\mathrm{e}, \mathrm{r}}$ and a low-boom target indicate how far the current configuration is from a threedimensional low-boom design, (2) the differences between $A_{\mathrm{e}, \mathrm{r}}$ and a low-boom target can be used to directly determine design regions and define design variables for low-boom shaping, and (3) finding an achievable lowboom target for $A_{\mathrm{e}, \mathrm{r}}$ is much easier than finding an achievable target for a mid-field $d p / p$.

A low-boom supersonic demonstrator concept for a cruise Mach number of 1.6 and a cruise weight of 30,000 lb was developed by matching $A_{\mathrm{e}, \mathrm{r}}$ to a low-boom target. The final design has a fully shaped ground signature with a perceived loudness value of 78.5 PLdB.

\section{Appendix}

The matrices for the reversed molecular relaxation and the reversed thermoviscous absorption in the iterative reverse propagation process are tridiagonal and are defined as follows:

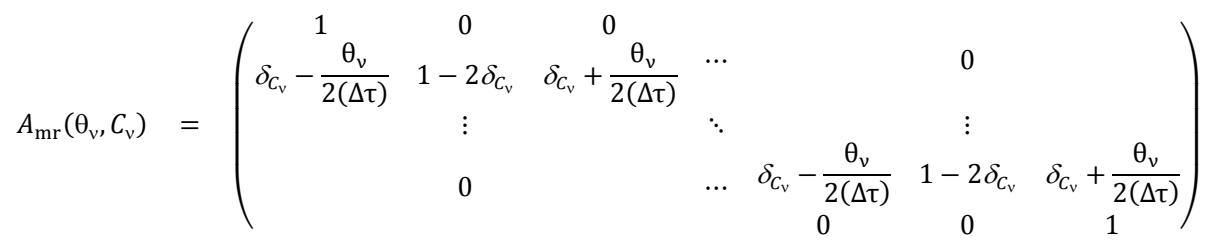

$$
\begin{aligned}
& B_{\mathrm{mr}}\left(\theta_{v}, C_{v}\right)=\left(\begin{array}{ccccccc}
1 & 0 & 0 & & & & \\
\beta_{C_{v}}-\frac{\theta_{v}}{2(\Delta \tau)} & 1-2 \beta_{C_{v}} & \beta_{C_{v}}+\frac{\theta_{v}}{2(\Delta \tau)} & \ldots & & 0 & \\
& \vdots & & \ddots & & \vdots & \\
& 0 & & \ldots & \beta_{C_{v}}-\frac{\theta_{v}}{2(\Delta \tau)} & 1-2 \beta_{c_{v}} & \beta_{C_{v}}+\frac{\theta_{v}}{2(\Delta \tau)}
\end{array}\right)
\end{aligned}
$$

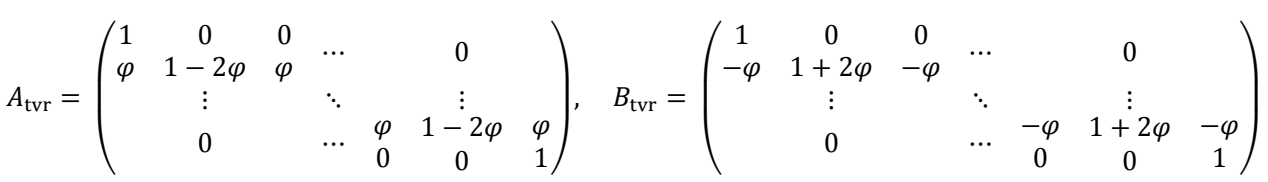

The constants in the above matrices are defined as

$$
\begin{gathered}
\delta_{C_{v}}=\frac{C_{v}\left(\sigma_{k}-\sigma_{k-1}\right)}{(\Delta \tau)^{2}} \cdot\left(\alpha-\frac{\varepsilon}{C_{v}\left(\sigma_{k}-\sigma_{k-1}\right)}\right) \\
\beta_{C_{v}}=\frac{C_{v}\left(\sigma_{k}-\sigma_{k-1}\right)}{(\Delta \tau)^{2}} \cdot\left(\alpha-1-\frac{\varepsilon}{C_{v}\left(\sigma_{k}-\sigma_{k-1}\right)}\right) \\
\varphi=\frac{\sigma_{k}-\sigma_{k-1}}{2 \Gamma(\Delta \tau)^{2}}\left(1-\frac{2 \varepsilon}{\sigma_{k}-\sigma_{k-1}}\right)
\end{gathered}
$$




\section{References}

1 "ModelCenter and Optimization Tools," Phoenix Integration, http://www.phoenix-int.com, [cited May 2011].

${ }^{2}$ Li, W., Shields, E., and Geiselhart, K., "A Mixed-Fidelity Approach for Design of Low-Boom Supersonic Aircraft," AIAA-2010-0845, January 2010. (Also accepted for publication in the J. of Aircraft.)

${ }^{3} \mathrm{Li}$, W. and Shields, E., "Generation of Parametric Equivalent-Area Targets for Design of Low-Boom Supersonic Concepts," AIAA-2011-462, January 2011.

${ }^{4} \mathrm{Li}$, W., Shields, E., and Le, D., "Interactive Inverse Design Optimization of Fuselage Shape for Low-Boom Supersonic Concepts," Journal of Aircraft, Vol. 45, No. 4, 2008, pp. 1381-1398.

${ }^{5}$ Nadarajah, S., Soucy, O., and Balloch, C., "Sonic Boom Reduction via Remote Inverse Adjoint Approach," AIAA2007-56, January 2007.

${ }^{6}$ Daumas, L., Dinh, Q., Kleinveld, S., and Rogé, G., "Automatic Shape Optimization Using Parametric CAD Applied to Sonic Boom Reduction," AIAA-2007-1919, April 2007.

${ }^{7}$ Choi, S., Alonso, J., Kroo, I., and Wintzer, M., "Multifidelity Design Optimization of Low-Boom Supersonic Jets," Journal of Aircraft, Vol. 45, No. 1, 2008, pp. 106-118.

${ }^{8}$ Yamashita, H., and Obayashi, S., "Numerical Investigation on Sonic Boom Reduction with Non-axisymmetric Body Shapes," AIAA-2008-59, January 2008.

${ }^{9}$ Chernyshev, S., Kiselev, A., and Vorotnikov, P., "Sonic Boom Minimization and Atmospheric Effects," AIAA2008-59, January 2008.

${ }^{10}$ Nemec, M., and Aftosmis, M., "Parallel Adjoint Framework for Aerodynamic Shape Optimization of ComponentBased Geometry," AIAA-2011-1249, January 2011.

${ }^{11}$ Rallabhandi, S., “Advanced Sonic Boom Prediction Using Augmented Burger's Equation,” AIAA-2011-1278, January 2011.

${ }^{12}$ Tantserev, E., "Backward Modeling of Thermal Convection: A New Numerical Approach Applied to Plume Reconstruction," URL: http://org.ntnu.no/geophys/pres/280208 2.ppt, [cited May 2011].

${ }^{13}$ Plotkin, K., "Review of Sonic Boom Theory," AIAA-1989-1105, April 1989.

${ }^{14}$ Seebass, R., and Argrow, B., "Sonic Boom Minimization Revisited," AIAA-1998-2956, June 1998.

${ }^{15}$ Aftosmis, M., "Cart3D Web Resource," URL: http://people.nas.nasa.gov/ aftosmis/cart3d/cart3Dhome.html, [cited May 2011].

${ }^{16}$ Ordaz, I., and Li, W., "Integration of Off-Track Sonic Boom Analysis in Conceptual Design of Supersonic Aircraft," AIAA-2011-464, January 2011. 